\title{
SHIPYARD CRANE MODELING METHODS
}

\author{
Pawel Piskur'), Piotr Szymak ${ }^{1)}$, \\ Bartosz Larzewski ${ }^{2}$ \\ 1) FUD Technologies Sp. z o.o. (FT) (Poland) \\ 2) Polish Naval Academy (Poland)
}

\begin{abstract}
The article discusses various crane mathematical modelling and simulation methodologies. The purpose of this study is to assess the effect of wind force on the dynamic of shipyard cranes, particularly hook movements in the horizontal plane. Appropriate simulation models are required to offer a robust control strategy that allows the crane to be remotely operated in windy circumstances. As a result, mathematical models based on differential equations for varying numbers of independent variables are compared to object-oriented, physical modelling model based on Matlab Simscape Multibody. The assumptions are explored, as well as the effect of the number of independent variables on model correctness.

Keywords: shipyard crane; nonlinear dynamic system; state space; transmittance; Simscape multibody simulation
\end{abstract}

\section{Introduction}

Shipyard cranes are commonly employed in industry to transfer huge cargoes. Nowadays, if the wind force is too strong it is impossible to operate shipyard cranes for more than a few days. Researches are being conducted in order to implement a new form of controller to mitigate the effects of a wind force. Although the study (Lee 2003) provides multiple control solutions to eliminate loading swing during crane movement, the analysis is provided for windless situations. The shipyard crane is a multi-input, multi-output nonlinear system. The wind impact is rarely considered while identifying all processes and relationships in an object. Furthermore, most simulation models consider the phenomena to be discrete in space, therefore the effects of crane hook form or number of ropes on system dynamics is not investigated.

The crane system's behaviour can be modelled in either the time or frequency domain. Following that, system properties such as rising time, overshoot, and settling time can be explored. Bode diagrams, Nichols charts, gain and phase margins, and pole and zero positions can all be used to optimise the control system unit. More advanced control algorithms, such as predictive control, can also be adapted. However, an exact mathematical model is required to make the tune 
control more reliable. The tuning control algorithm approach is determined by whether linear or nonlinear interactions are described in the model.

The following stages were completed to construct an adequate mathematical model based on the technique described in the study (Piskur et al. 2009). The definition and specification of the item and its environment were realised at the start. The accuracy and extent of validity of the model were determined based on its designation. The assumptions and simplifications should be defined for each phenomenon. Choosing whether the phenomenon is spatially spread or discrete. If distributed, and given continuity, symmetry, and the number of space dimensions (1D, 2D or 3D). The next stage is to define mathematical relationships and decide how to solve the model. According to the rule, it consists of nonlinear partial or ordinary equations, necessitating the use of a computer programme.

Matlab, a popular software with ordinary differential equation solvers, is widely used. Ordinary differential equations can be solved analytically as well as using the Matlab-Simulink block language. The number of ordinary equations is proportional to the space dimension. If the crane is analysed as a continuous in space, the Finite Element Method should be used; however, this method is time-consuming and difficult to design for complicated systems.

The article goes over different simulation models based on environmental assumptions and the number of degrees of freedom. Because of the challenges in analysing the impact of crane form on shipyard crane dynamics, the object-oriented method is given as a Simscape Multibody toolbox in Matlab software. In the case of control system unit design, extra efforts are taken to check the accuracy of the simulation model. The key distinction between current simulation models is that a new study for shipyard cranes with many ropes can be offered. Furthermore, the impact on wind force can be investigated, and as a result, the crane geometry can be incorporated in the study.

Conclusions are illustrated at the end of the work, with connections to achieved results and alternative ways of modelling crane systems.

The study's goal is to see if multibody simulation techniques can be used for shipyard crane dynamic simulation.

\section{Mathematical models of a shipyard crane}

The crane system's general mathematical description is based on the Lagrangian approach (1). To characterise the dynamic behaviour of the system, the EulerLagrange formulation should be addressed (1).

$$
\frac{d}{d t} \frac{\partial L}{\partial \dot{q}_{k}}-\frac{\partial L}{\partial q_{k}}=0 ; k=1, \ldots, n
$$

where:

$\mathrm{n}$ - is the degree of freedom (DOF) of the system;

$\mathrm{q} 1, \ldots, \mathrm{qn}$ - signifies generalized coordinates. 
The difference between the kinetic (KE) and potential (PE) energy of the nDOF system is represented by the Lagrangian $\mathrm{L}=\mathrm{KE}-\mathrm{PE}$. The dynamics of the $\mathrm{n}$-DOF system are characterised by a set of $\mathrm{n}$ connected second-order differential equations, according to Newton's law. The second-order equations are then transformed to a set of first-order equations in order to be numerically solved. To obtain the continuous-time linear model, a strategy with a Jacobian linearization of the nonlinear system of equations around the linked system equilibrium point is described. Model linearization is useful for configuring control system algorithms.

A state-space model of the continuous-time form can be used to describe both linear and nonlinear system dynamics (2):

$$
\begin{aligned}
& \frac{d x}{d t}=A x+B u \\
& y=C x+D u
\end{aligned}
$$

or discrete-time form (3):

$$
\begin{aligned}
& x[n+1]=A x[n]+B u[n] \\
& y[n]=C x[n]+D u[n]
\end{aligned}
$$

where:

$\mathrm{A}, \mathrm{B}, \mathrm{C}$, and D - are appropriate-dimension matrices, $\mathrm{X}$ - is the state vector,

$\mathrm{u}$ and $\mathrm{y}-$ are the input and output vectors,

$x[n], u[n]$, and $y[n]-$ are the state, input, and output vectors for the $n$-th sample.

The transfer function can be provided for state-space models based on linear differential equations. The continuous-time transfer function can be represented as the ratio (4) for a single input and single output.

$$
G(s)=\frac{N(s)}{D(s)}=\frac{b_{m} s^{m}+\cdots+b_{1} s+b_{0}}{a_{n} s^{n}+\cdots+a_{2} s^{2}+a_{1} s+a_{0}}
$$

where:

$\mathrm{N}(\mathrm{s})$ - numerator polynomials,

$\mathrm{D}(\mathrm{s})$ - denominator polynomials.

Because the transfer function form is unique, converting from a state-space form to a transfer function is simple. The process of converting from transfer function to state space is more difficult, owing to the fact that various state-space forms define a system.

The final method shown in this research is object-oriented model-based design using the Matlab Simscape Multibody toolkit. It enables the creation of a multi-body 
crane model with varying degrees of freedom. Furthermore, the simulation model provides a fast overview of how it works in terms of archiving all interesting dynamic data. There is also the option to perform simulation analysis using a different control algorithm. However, various parameters and coefficients, such as friction coefficients, are required. As a result, two laboratory cranes were employed for mathematical model testing and identifying unknown parameters (Omar et al. 2004).

\section{Dynamic mathematical model for crane dynamic in one plane}

The simplest basic crane model analysis has one degree of freedom, one firm rope, and a distinct mass in space (Figure 1). Newton's second rule states that for a crane block dynamic in one plane, the mass times acceleration must equal the component of gravity's force (5):

$$
\frac{d^{2} \theta}{d t^{2}}+\frac{g}{r} \sin (\theta)=0
$$

where:

$\theta-$ is the angle of the pulley rope deviation from the direction of gravity;

$\mathrm{r}-\mathrm{a}$ rope length;

$\mathrm{g}-$ an acceleration due to gravity.

$\mathrm{t}-\mathrm{a}$ time (independent variable).

For the assumption of a small angle, the nonlinear second-order ordinary differential equation (5) can be linearized:

$$
\sin (\theta) \approx \theta
$$

Then, the equation (6) can be formulated as formula (7):

$$
\frac{d^{2} \theta}{d t^{2}}+\frac{g}{r} \theta=0
$$

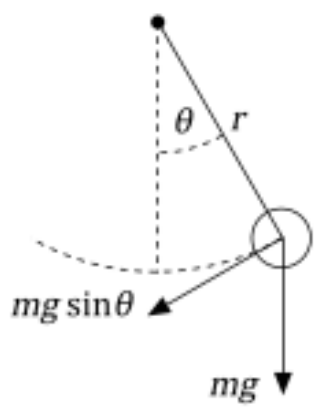

Figure 1. The simplest model of a crane with one DOF 
During the investigation, the practical relationship employed was the period proportionate to the square root of the pendulum's length (8):

$$
T=\frac{2 \pi}{\omega_{0}}=2 \pi \sqrt{\frac{r}{g}}
$$

For the linear equation, the period is independent of mass and initial condition. These dependencies can be utilised to validate the simulation model based on a mathematical model and to calculate the resonance frequency.

If the hook mass cannot be omitted and its dimensions are not negligible due to the air damping force, the following equation can be used:

$$
m r^{2} \frac{d^{2} \theta}{d t^{2}}+r g \sin (\theta)=F_{d a m p}
$$

where:

$\mathrm{m}-$ is the a mass of the hook;

F_dump - is the total of damping forces caused by friction at the pivot point connection and air damping force.

Taking the damping force into account, equation (9) can be rewritten as (10):

$$
m r^{2} \frac{d^{2} \theta}{d t^{2}}+r g \sin (\theta)=k r \frac{d \theta}{d t}+F_{D}
$$

where:

$\mathrm{k}$ - the friction coefficient at the pivot point;

F_D - the force from air acting on the hook geometry, described by expression:

$$
F_{D}=\frac{1}{2} \rho v^{2} C_{D} A
$$

where:

$\rho$ - the air density $1.2\left[\mathrm{~kg} / \mathrm{m}^{3}\right]$;

$\mathrm{v}$ - the speed of a wind $[\mathrm{m} / \mathrm{s}]$,

$\mathrm{C}_{\mathrm{D}}$ - the drag coefficient [-],

$\mathrm{A}$ - the block surface $\left[\mathrm{m}^{2}\right]$.

If a shipyard crane is to be used in windy conditions, the wind speed must be measured. Based on the foregoing equations, a non-linear simulation model of a crane (Figure 2) was created, with the unknown values of the damping force and wind effect as input variables $\mathrm{T}$. 


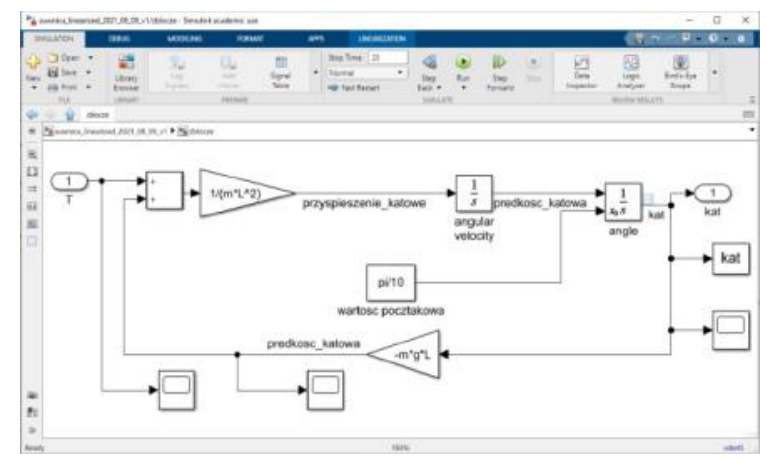

Figure 2. A simulation model of a shipyard crane in one plane with a moment of wind force acting on the hook

The value of the external force is calculated by taking the length of the pulley ropes, i.e. the arm on which the force operates, and the block mass into consideration.

The following form of transmittance can be defined if the aim is to assess the wind's impact on the dynamics of the gantry block:

$$
G(s)=\frac{\theta(s)}{v(s)},
$$

The input signal is the wind speed and the output signal is the change of the block position in the horizontal plane. Such an analysis will allow for predictive control of the gantry, as a function of changing the wind velocity. The purpose of the control is to prevent gusts of wind, especially those with the frequency of changes coinciding with the resonant frequency.

If the pivot point is mounted on the cart then the mutual interaction between hook and cart should be considered. In addition, the friction between the cart and the trolleys are difficult to established. An example of procedure for friction coefficient identification for laboratory crane is presented in the paper (Omar et al. 2004).

\section{Complete nonlinear crane model with one rope}

Complete nonlinear model of the crane with constant rope length is described with using eight state equations (Aksjonov et al. 2015; Aksjonov et al. 2016). The same crane system with varying rope length is described using ten nonlinear state equations (Ma et al. 2018). But the model is verified for a single rope sling. There was also assumption like viscous damping coefficient, mass moment of inertia and rail motors are directly cancelled by the controller.

The number of ropes has impact on crane block stabilization. It is difficult to expand the equation which includes some ropes impacted on the crane dynamics. Also ropes parameters have impact on the hook movements in horizontal plane, 
that is why decision was made to prepare simulation model in Simscape Multibody toolbox ${ }^{1)}$.

\section{Crane simulation model in Simscape Multibody}

Simscape Multibody provides a multi-body dynamic simulation environment of a mechanical system in three-dimensional space. In Figure 3 the subsystem of mechanical crane is presented. The simulation model assembles blocks representing bodies, joints and force elements into schematic (Figure 4). Simscape Multibody formulates and solves the equations of motion for the complete mechanical system.

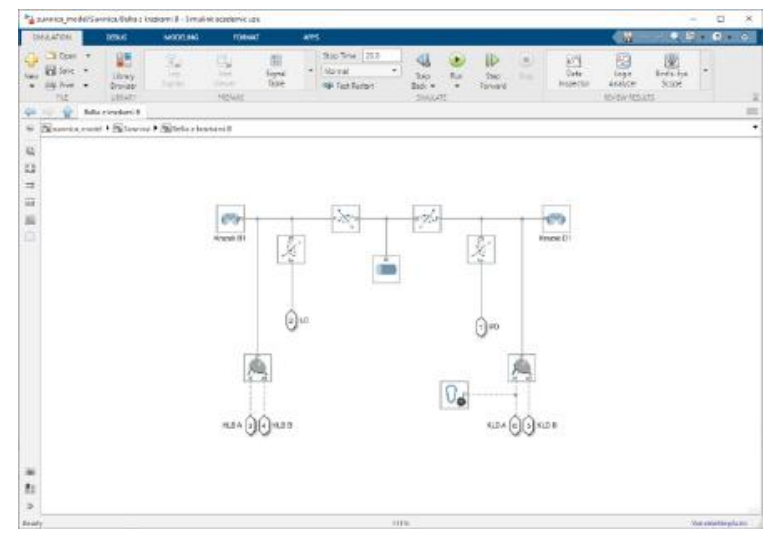

Figure 3. The subsystem of the 3D crane simulation model

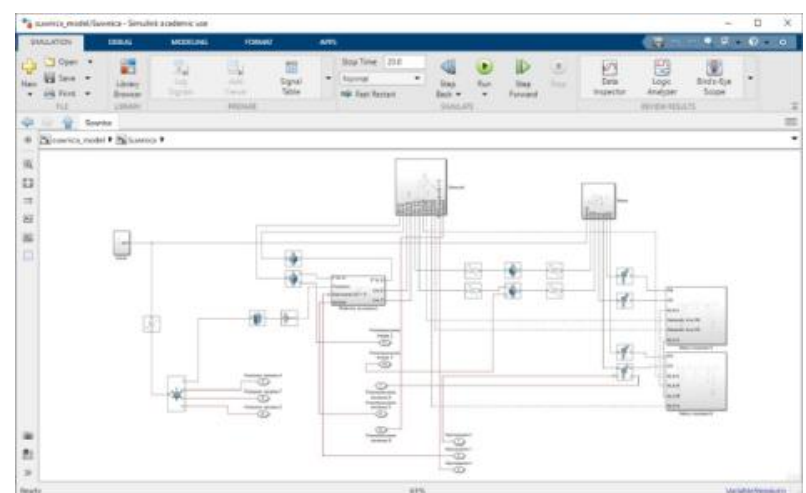

Figure 4. The scheme of 3D crane simulation model

The complete CAD assemblies, including all masses, inertias, joints, constraints, and $3 \mathrm{D}$ geometry, can be imported into the simulation model. Then, the multibody 
system (Figure 5) can be used to simulate the output signals as a function of input signals.

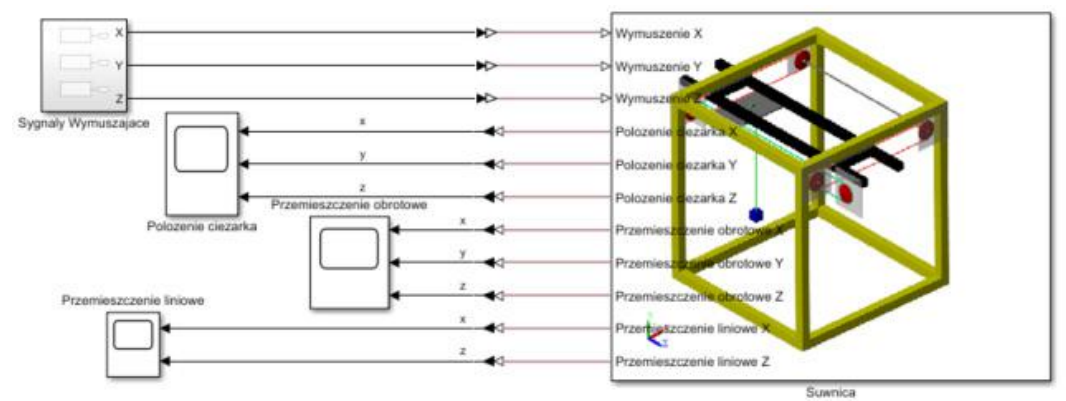

Figure 5. 3D crane simulation model with control and observation signals

An automatically generated $3 \mathrm{D}$ animation lets visualize the system dynamics (Figure 6).

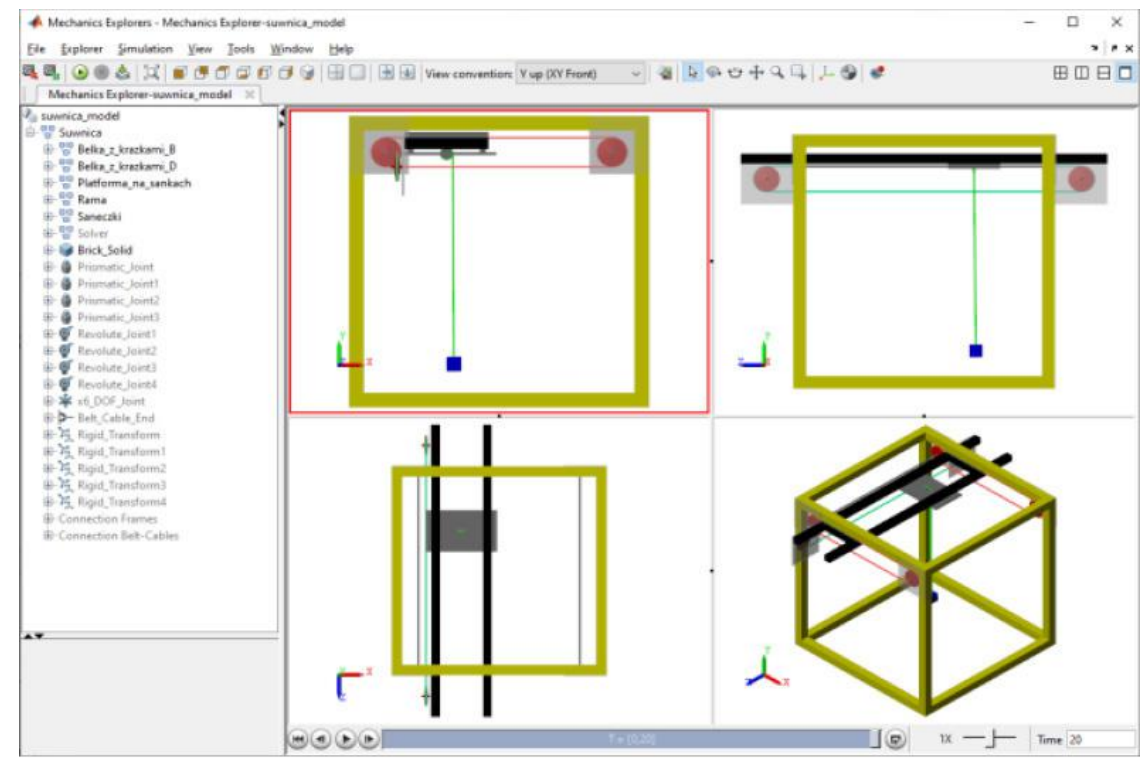

Figure 6. 3D Crane simulation model visualization in Simscape Multibody

The simulation model (Figure 7) is based on the laboratory crane (Figure 8) manufactured by INTECO $^{2)}$ company by one rope. 


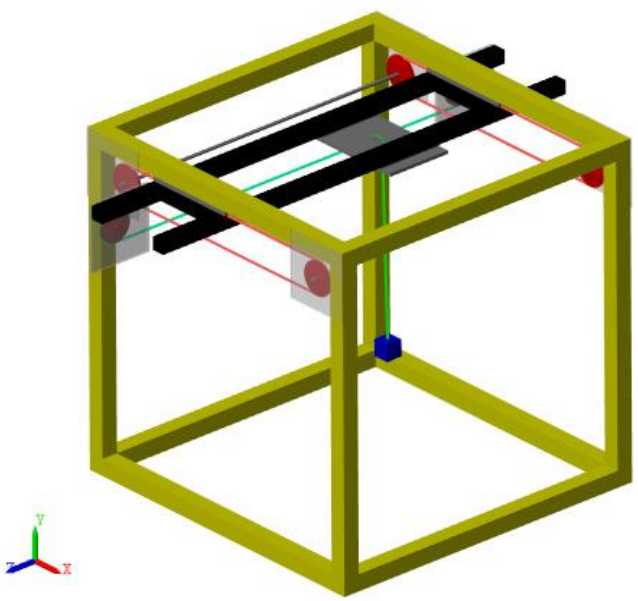

Figure 7. The 3D crane simulation model by one rope

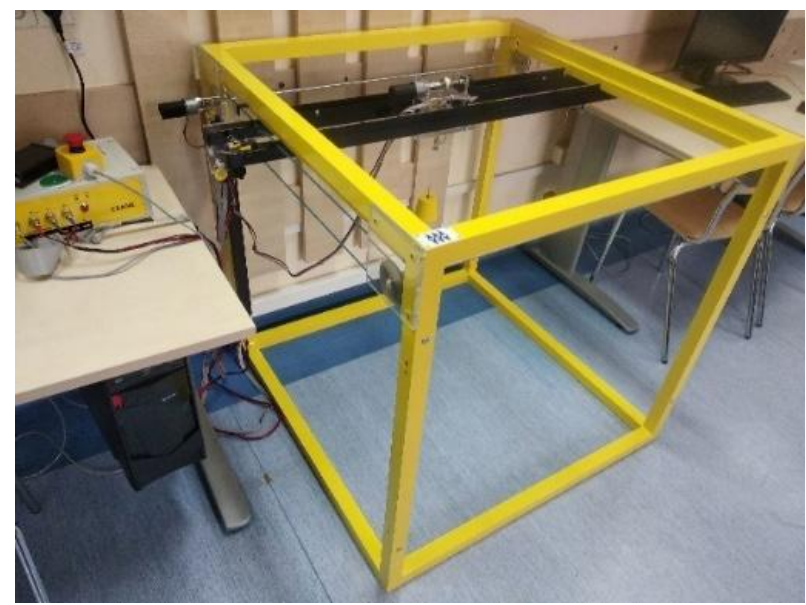

Figure 8. The crane laboratory model by one rope

After the crane by one rope model validation, the second laboratory crane with multiple ropes was taken under consideration. In Figure 9 two images are presented with the crane block in top and the lower positions. This is the laboratory crane designed for verification of multi ropes system impact on the block crane dynamics. It is also possible to investigate the required friction coefficient in the simulation model, as shown in Figure 10. 
a)

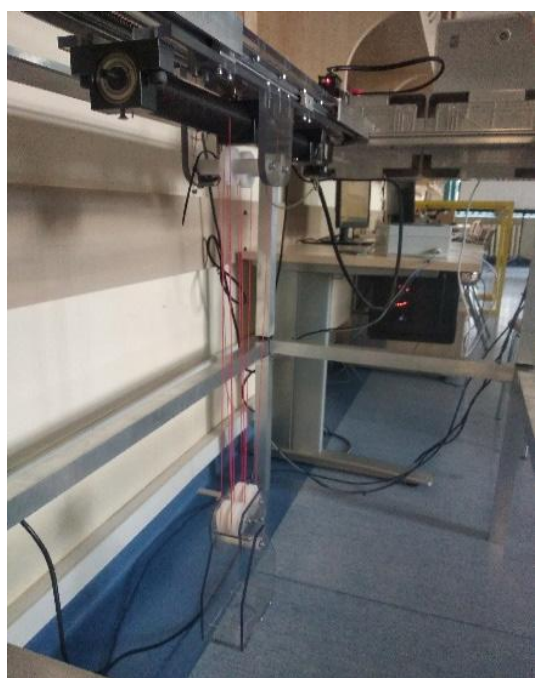

b)

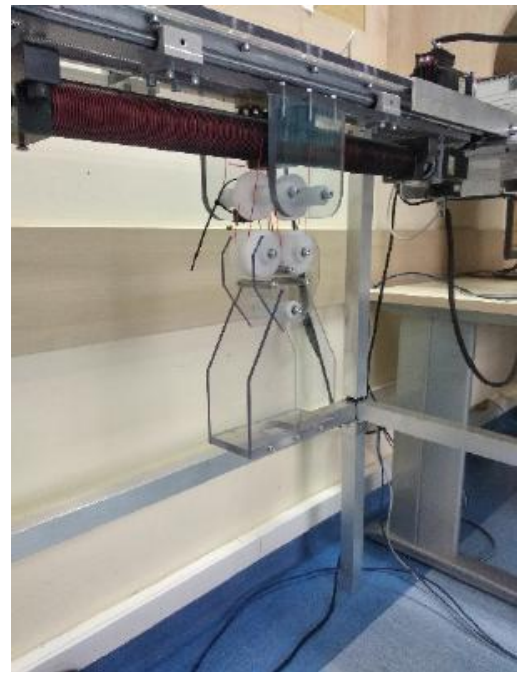

Figure 9. The crane laboratory model by multiple ropes:

a) in the lower position, b) in the top position

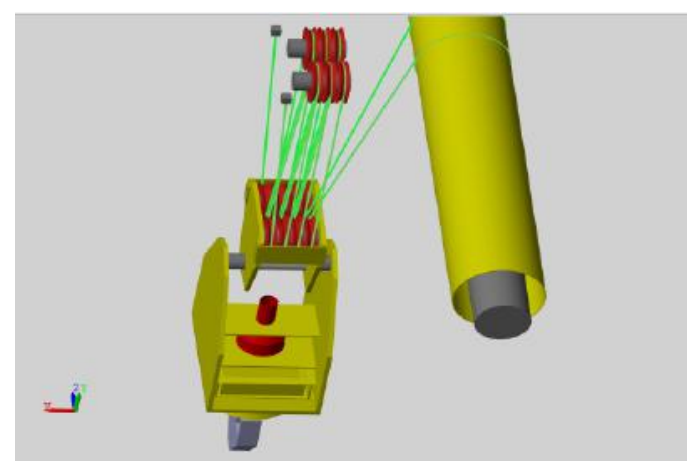

Figure 10. Crane block simulation model with multiple ropes

After creating a simulation model for one cable and multi-rope crane system, an analysis of the impact of construction or material data on the crane dynamics can be performed. Also, the wind impact on the block can be analysed and controller can be design to reduce the oscillation of the crane block. This will ensure the safety working condition for shipyard crane operations during windy weather condition.

\section{Conclusions}

In this paper, different types of shipyard crane models were discussed with the number of independent variables impact on usefulness model for specific analysis. 
The proper simulation model is achieved from adequate mathematical model with the definition and specification of the object and its environment.

The comparison of simulation models shows that Simscape Multibody technics can be adopted for control designing process. A 3D animation visualizes the system dynamics in a convenient way. This method is far more secure and efficient than testing on hardware prototypes. As a result, tasks like designing control systems and optimising system level performance can be completed more quickly.

Simscape Multibody formulates and solves the equations of motion for the complete mechanical system. The three dimensional geometry can be defined or imported into dynamic model and then controller parameters can be tuned.

Future research includes a controller analysis as well as the effect of the number of ropes on the stabilization of the crane hook in windy conditions.

\section{Acknowledgments}

The results presented in the paper have been obtained within the development project no POIR.01.02.00-00-0017/18 financed by the Polish National Centre of Research and Development in 2019 - 2022 years.

\section{NOTES}

1. https://www.mathworks.com/help/control/ug/state-space-models.html

2. http://www.inteco.com.pl/products/3d-crane/

\section{REFERENCES}

Aksjonov, A., Vodovozov, V. \& E. Petlenkov, 2015. Sensorless control of the three-dimensional crane using the Euler-Lagrange approach with a built-in state-spaee model. In: 56th International Scientific Conference on Power and Electrical Engineering of Riga Technical University (RTUCON), 1 - 4. Riga, Latvia. Available from: doi: 10.1109/RTUCON.2015.7343138.

Aksjonov, A., Vodovozov, V. \& Petlenkov, E., 2016. Three-Dimensional Crane Modelling and Control Using Euler-Lagrange State-Space Approach and Anti-Swing Fuzzy Logic. Electrical, Control and Communication Engineering. 9(1), 5 - 13. Available from: https://doi.org/10.1515/ ecce-2015-0006

Khatamianfar, A., Savkin, A., 2019. Real-Time Robust and Optimized Control of a 3D Overhead Crane System. Sensors. 19(15), 3429. Available from: https://doi.org/10.3390/s19153429

Lee, Ho-Hoon, 2003. A new approach for the anti-swing control of overhead cranes with high-speed load hoisting. International Journal of Control 76, 1493-1499. Available from: doi: 10.1080/00207170310001604954. 
Ma, X., Bao, H., 2018. An Anti-Swing Closed-Loop Control Strategy for Overhead Cranes. Appl. Sci. 8, 1463.

Omar, HM, Nayfeh, AH., 2004. Gain Scheduling Feedback Control of Tower Cranes with Friction Compensation. Journal of Vibration and Control 10(2), 269 - 289. Available from: doi: 10.1177/1077546304035610

Piskur, P., Tarnowski, W. \& Just, K., 2009, June. Model Of The Electromagnetic Linear Actuator For Optimization Purposes. In: ECMS, 708713. Available from: doi: $10.7148 / 2009-0708-0713$

Zhang, Z., Chen, D. \& Feng, M., 2008. Dynamics model and dynamic simulation of overhead crane load swing systems based on the ADAMS. In: 9th International Conference on Computer-Aided Industrial Design and Conceptual Design, 484 - 487. Available from: doi: 10.1109/CAIDCD.2008.4730616.

$\triangle$ Pawel Piskur

ORCID iD: 0000-0002-8823-4316

FUD Technologies Sp. z o.o. Gdynia, Poland

E-mail: p.piskur@amw.gdynia.pl

$\triangle$ Piotr Szymak

ORCID iD: 0000-0002-4714-6192

FUD Technologies Sp. z o.o.

Gdynia, Poland

E-mail: p.szymak@amw.gdynia.pl

Bartosz Larzewski

Polish Naval Academy Gdynia, Poland

E-mail: 322855@edu.amw.gdynia.pl 